\title{
CIGRE-Session 2004
}

Die 40. Tagung der CIGRE (International Council on Large Electric Systems) fand heuer vom 29. August bis 3. September wie gewohnt in Paris statt.

Über 2000 Teilnehmern aus 80 Ländern (Österreich war mit 20 Teilnehmern vertreten) bot sich dabei die Gelegenheit, Fragen und Lösungsvorschläge zu Problemen in der Elektrotechnik zu diskutieren. Aufgrund der großen Zahl heute aktueller technischer und wirtschaftlicher Schwerpunkte wurden erstmals 45 Vorzugsthemen ausgewählt. Dazu wurden 344 Berichte vorgelegt, die in voller Länge (auf CD-ROM) bei der CIGRE erhältlich sind. Auch drei Beiträge österreichischer Autoren bzw. Koautoren fanden Beachtung, die Sie im Folgenden abgedruckt finden:

- „An algorithm for the three-pole controlled auto-reclosing of shunt compensated transmission lines with an optimization for the second and third pole" von H.M. Muhr, S. Pack (Österreich), G. Pilz, P. Schegner, C. Wallner (Deutschland)

- "Corrosion protection of steel towers and camouflage of lines using the DUPLEX-system" von $\mathrm{H}$. Lugschitz, A. Ernst, T. Gros (Österreich)

- "High voltage series reactors for load flow control" von $\mathrm{K}$. Papp, G. Christiner, H. Popelka, (Österreich), M. Schwan (Deutschland)

Neben den etwa fünfstündigen Plenarveranstaltungen der 16 Studienkomitees wurden nicht weniger als 114 weitere Sitzungen der Studienkomitee-Arbeitsgruppen abgehalten.

Gleichzeitig fand - ergänzend zu den Vorträgen und Diskussionen - wieder die CIGRE Expo statt, an der sich 84 Aussteller beteiligten. Dabei hatten die Tagungsteilnehmer Gelegenheit, einen Überblick über die besten heute zur Verfügung stehenden Produkte und Technologien zu gewinnen.

Darüber hinaus boten sich auch während der heurigen Tagung, vor allem anlässlich der Abendeinladung durch das französische Nationalkomitee, viele Möglichkeiten zu Gesprächen und zum persönlichen Kennenlernen.

Im Rahmen der Session fand die ordentliche Vollversammlung 2004 der CIGRE statt. Dabei wurden die Mitglieder des

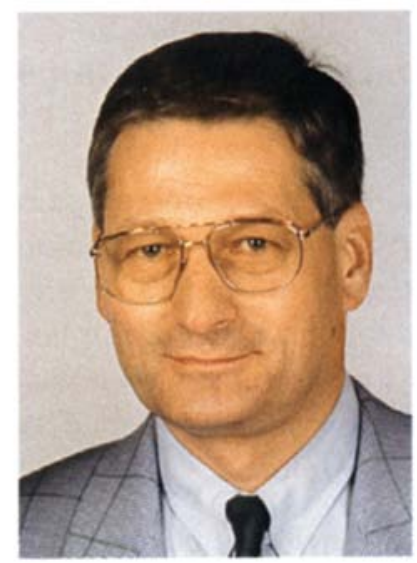

Peter Morawek

"Administrative Council" gewählt. Wie schon in der Vergangenheit wird Österreich auch während der nächsten Jahre wieder durch ein Mitglied in diesem Gremium vertreten sein.

Da die Funktionsperiode des amtierenden CIGRE-Präsidenten David Croft (Präsident Transgrid, Australien) zu Ende ging, wurde aus einem Wahlvorschlag für zwei Kandidaten schließlich Yves Filion (Präsident Hydro Québec, Canada) zum neuen Präsidenten gewählt.

Erstmals in ihrer 83-jährigen Geschichte hat die CIGRE über 5000 Mitglieder und 53 Nationalkomitees in 80 Ländern zu verzeichnen.

Das österreichische Nationalkomitee umfasst heute 26 Einzel- und 23 Kollektivmitglieder. Österreichische Experten arbeiten in neun Studienkomitees und in einer Vielzahl von Arbeitsgruppen der CIGRE.

\section{VDir. Dipl.-Ing. Dr. Peter Morawek OVE}

Vorsitzender des Österreichischen Nationalkomitees der CIGRE 